\title{
The Simplex Structure of the Classical States of the Quantum Harmonic Oscillator
}

\author{
A. Bach ${ }^{1}$ and U. Lüxmann-Ellinghaus ${ }^{2}$ \\ ${ }^{1}$ Institut für Theoretische Physik I, Universität Münster, D-4400 Münster, Federal Republic of \\ Germany \\ ${ }^{2}$ Fachbereich Mathematik, Universität Dortmund, D-4600 Dortmund 50, Federal Republic of \\ Germany
}

\begin{abstract}
It is shown that the convex set of classical states of the quantum harmonic oscillator is a simplex generated as the closed convex hull of the coherent states in the weak topology of the Banach space of trace class operators.
\end{abstract}

\section{Introduction}

Let $\psi(x, y) \in H$, where $(x, y) \in \mathbb{R}^{2}$, denote a coherent state vector of the onedimensional quantum harmonic oscillator

$$
\psi(x, y)=\exp \left(-\frac{x^{2}+y^{2}}{2}\right) \sum_{n=0}^{\infty} \frac{(x+i y)^{n}}{\sqrt{n !}} \varphi_{n} .
$$

Here $\varphi_{n}, n \in \mathbb{Z}_{+}$, are the eigenvectors of the number operator which constitute a complete orthonormal set of the Hilbert space $H$ under consideration.

In the following $L^{1}(H)$ denotes the Banach space of trace class operators on $H$ equipped with the trace norm $\|\cdot\|_{1}$, and $S(H)$ is the set of statistical operators

$$
S(H)=\left\{W \in L^{1}(H) ; \quad W \geqq 0, \operatorname{tr}(W)=1\right\} .
$$

A state on $L(H)$, the Banach space of bounded linear operators on $H$, is called a coherent state if it can be identified with a statistical operator of the form

$$
P(x, y)=(\psi(x, y), \cdot) \psi(x, y)
$$

where $(x, y) \in \mathbb{R}^{2}$ and $\psi(x, y)$ is defined in (1). For information concerning the physical and mathematical properties of coherent states we refer to [1,2]. There the $P$ representation of a statistical operator $W$ is introduced as an integral representation in terms of coherent states

$$
W=\int_{\mathbb{R}^{2}} d x d y p(x, y) P(x, y),
$$

where $p(x, y)$ is called a quasi-probability insofar as it is discerned from a probability 
density on $\mathbb{R}^{2}$ by the fact that it is admitted that

i) $p(x, y)$ takes negative values,

ii) $p(x, y)$ is a distribution.

Besides the fact that the integral in (4) is defined in a weak sense only, we see the shortcomings of this approach to an integral representation of statistical operators in the application of quasi-probabilities which are unnatural from a probabilistic point of view.

In this note we attack the representation problem by means of a different strategy. We focus on representations of the form (cf. [3])

$$
W=\int d \mu(x, y) P(x, y)
$$

(which are rigorously defined below) where $\mu$ is a probability measure on $\mathbb{R}^{2}$, i.e. $\mu \in M_{+}^{1}\left(\mathbb{R}^{2}\right)$. Obviously, in this approach not very statistical operator is representable. On the other hand it turns out that the set of statistical operators which are representable has $\mathrm{a}$, in the sense of classical statistical mechanics, typical classical structure: it is a simplex. Here a convex set is defined to be a simplex provided the cone generated by it is a lattice in its own order.

\section{Classical States}

Our aim is to give a rigorous meaning to the integral (5). To this end we recall that due to the fact that $(x, y) \rightarrow \psi(x, y)$ is continuous (cf. [2]) the mapping $\Phi: \mathbb{R}^{2} \rightarrow L^{1}(H)$ defined by

$$
\Phi(x, y)=P(x, y)
$$

is continuous, too. This implies that the range of $\Phi$ is a separable subset of $L^{1}(H)$ and, in turn, that $\Phi$ is strongly measurable. Moreover, by virtue of $\|P(x, y)\|_{1}=1$, for any $\mu \in M_{+}^{1}\left(\mathbb{R}^{2}\right)$,

$$
\int d \mu(x, y)\|P(x, y)\|_{1}=1
$$

holds. These facts entail that $\Phi$ is Bochner integrable with respect to any $\mu \in M_{+}^{1}\left(\mathbb{R}^{2}\right)$ (cf. [4]). Obviously, for any $\mu \in M_{+}^{1}\left(\mathbb{R}^{2}\right)$ the integral $\int d \mu(x, y) P(x, y) \in S(H)$ such that the following definition makes sense.

Definition. Whenever there exists a probability measure $\mu \in M_{+}^{1}\left(\mathbb{R}^{2}\right)$ such that a statistical operator $W \in S(H)$ is representable as a Bochner integral via $W=$ $\int d \mu(x, y) P(x, y)$, the state on $L(H)$ determined by $W$ is called a classical state.

It is evident that the set of classical states which we denote by $\operatorname{CS}(H)$ is a convex subset of the convex set $S(H)$ and it is our goal to show that $C S(H)$ is a simplex contained in $S(H)$. To this end we need some properties of the mapping $D: M_{+}^{1}\left(\mathbb{R}^{2}\right) \rightarrow C S(H)$ defined by

$$
D(\mu)=\int d \mu(x, y) P(x, y) .
$$

Let us introduce for all $\mu \in M_{+}^{1}\left(\mathbb{R}^{2}\right)$ a positive bounded measure $v[\mu] \in M_{+}^{b}\left(\mathbb{R}^{2}\right)$ by

$$
\frac{d v[\mu]}{d \mu}(x, y)=\exp \left(-\left(x^{2}+y^{2}\right)\right),
$$


so that $v[\mu]\left(\mathbb{R}^{2}\right) \leqq 1$, and denote the characteristic function of $v[\mu]$ by $\hat{v}[\mu]$,

$$
\hat{v}[\mu](x, y)=\int d v[\mu]\left(x^{\prime}, y^{\prime}\right) \exp \left(i x x^{\prime}+i y y^{\prime}\right) .
$$

For any $(x, y) \in \mathbb{R}^{2}$ and any $\mu \in M_{+}^{1}\left(\mathbb{R}^{2}\right)$ we have, by the properties of Bochner integrals,

$$
\begin{aligned}
(\psi(x, y), D(\mu) \psi(-x,-y)) & =\int d \mu\left(x^{\prime}, y^{\prime}\right)\left(\psi(x, y), P\left(x^{\prime}, y^{\prime}\right) \psi(-x,-y)\right) \\
& =\int d \mu\left(x^{\prime}, y^{\prime}\right)\left(\psi(x, y), \psi\left(x^{\prime}, y^{\prime}\right)\right)\left(\psi\left(x^{\prime}, y^{\prime}\right), \psi(-x,-y)\right) .
\end{aligned}
$$

Taking into account Eq. (1) this yields for any $(x, y) \in \mathbb{R}^{2}$ and any $\mu \in M_{+}^{1}\left(\mathbb{R}^{2}\right)$,

$$
\begin{aligned}
& (\psi(x, y), D(\mu) \psi(-x,-y)) \\
& \quad=\int d \mu\left(x^{\prime}, y^{\prime}\right) \exp \left\{-\left(x^{2}+y^{2}\right)-\left(x^{\prime 2}+y^{\prime 2}\right)\right\} \exp \left\{i(-2 y) x^{\prime}+i(2 x) y^{\prime}\right\} \\
& \quad=\exp \left\{-\left(x^{2}+y^{2}\right)\right\} \hat{v}[\mu](-2 y, 2 x) .
\end{aligned}
$$

Next we associate with any $W \in S(H)$ a mapping $w: \mathbb{R}^{2} \rightarrow \mathbb{R}$ defined by

$$
w(x, y)=(\psi(x, y), W \psi(x, y)) .
$$

Obviously, for all $(x, y) \in \mathbb{R}^{2}$ we have $0 \leqq w(x, y) \leqq 1$ and $w \in C_{b}\left(\mathbb{R}^{2} ; \mathbb{R}\right)$ for any $W \in S(H)$. Due to the fact that the coherent state vectors are a total set in $H$ (cf. e.g. [3]) $w_{1}=w_{2}$ implies $W_{1}=W_{2}$. Moreover, since

$$
\int d x d y \frac{1}{\pi} w(x, y)=1
$$

holds (cf. e.g. [2]), $(1 / \pi) w$ can be considered as a probability density with respect to the Lebesgue measure $\lambda^{2}$ on $\mathbb{R}^{2}$. This fact allows us to associate with any $W \in S(H)$ a uniquely determined element $\rho_{W} \in M_{+}^{1}\left(\mathbb{R}^{2}\right)$ defined such that

$$
\frac{d \rho_{W}}{d \lambda^{2}}(x, y)=\frac{1}{\pi} w(x, y)
$$

Let us denote by $\gamma, \gamma \in M_{+}^{1}\left(\mathbb{R}^{2}\right)$, the probability measure with density

$$
\frac{d \gamma}{d \lambda^{2}}(x, y)=g(x, y)=\frac{1}{\pi} \exp \left(-\left(x^{2}+y^{2}\right)\right)
$$

and characteristic function

$$
\hat{\gamma}(x, y)=\exp \left\{-\frac{x^{2}+y^{2}}{4}\right\} .
$$

For any $W \in S(H)$ we define the quasicharacteristic function $\hat{\sigma}_{W}$ of $W$ by $\hat{\sigma}_{W}: \mathbb{R}^{2} \rightarrow \mathbb{C}$,

$$
\hat{\sigma}_{W}(x, y)=\exp \left(\frac{x^{2}+y^{2}}{4}\right) \hat{\rho}_{W}(x, y) .
$$

Obviously, $\hat{\sigma}_{W}$ is continuous and $\hat{\sigma}_{W}(0,0)=1$ holds.

With these prerequisites we state our first lemma. 
Lemma 1. For $W \in S(H)$ the quasicharacteristic function $\hat{\sigma}_{W}$ defined by Eq. (18) is a characteristic function of an element of $M_{+}^{1}\left(\mathbb{R}^{2}\right)$ if and only if $W \in C S(H)$. Moreover, for $D(\mu) \in C S(\mathrm{H})$

$$
\hat{\sigma}_{D(\mu)}=\hat{\mu}
$$

holds.

Proof.

i) Assume $W \in C S(H)$. Then there exists a $\mu \in M_{+}^{1}\left(\mathbb{R}^{2}\right)$ such that $W=D(\mu)$ and $w=d[\mu]$. But for any $\mu \in M_{+}^{1}\left(\mathbb{R}^{2}\right)$,

$$
\frac{1}{\pi} d[\mu](x, y)=(g * \mu)(x, y)
$$

holds for all $(x, y) \in \mathbb{R}^{2}$, where $*$ denotes the convolution.

Fourier transformation yields

$$
\hat{\rho}_{D(\mu)}(x, y)=\hat{\gamma}(x, y) \hat{\mu}(x, y)
$$

for all $\mu \in M_{+}^{1}\left(\mathbb{R}^{2}\right)$ and all $(x, y) \in \mathbb{R}^{2}$. From the definition (18) we have

$$
\hat{\sigma}_{W}(x, y)=\hat{\sigma}_{D(\mu)}(x, y)=(\hat{\gamma}(x, y))^{-1} \hat{\rho}_{D(\mu)}(x, y) .
$$

Comparing this with (21), the first part of the assertion and Eq. (19) follow.

ii) Assume $\hat{\sigma}_{W}$ is a characteristic function. Then there exists an uniquely determined probability measure $\mu_{W} \in M_{+}^{1}\left(\mathbb{R}^{2}\right)$ such that $\hat{\sigma}_{W}=\hat{\mu}_{W}$. It remains to be proved that $D\left(\mu_{W}\right)=W$. But by the assumption

$$
\hat{\rho}_{W}(x, y)=\hat{\gamma}(x, y) \hat{\sigma}_{W}(x, y)=\hat{\gamma}(x, y) \hat{\mu}_{W}(x, y),
$$

and by (19)

$$
\hat{\rho}_{D\left(\mu_{W}\right)}(x, y)=\hat{\gamma}(x, y) \hat{\mu}_{W}(x, y) .
$$

Therefore we have

$$
\hat{\rho}_{W}(x, y)=\hat{\rho}_{D\left(\mu_{W}\right)}(x, y)
$$

for all $(x, y) \in \mathbb{R}^{2}$. This implies

$$
\frac{1}{\pi} w(x, y)=\frac{1}{\pi} d\left[\mu_{W}\right](x, y)
$$

for all $(x, y) \in \mathbb{R}^{2}$ since both functions are continuous.

This ends the proof of the lemma.

Our conclusion is as follows. Whereas it is possible to associate with any statistical operator $W \in S(H)$ a probability measure, namely $\rho_{W}$, Lemma 1 shows that only for the classical states it is possible to define another characteristic probability measure in terms of $\hat{\sigma}_{W}$. And this one is just the mixing measure in the integral representation of these states. 


\section{The Simplex of Classical States}

To prove the simplex structure of $C S(H)$ we need a preliminary lemma.

Lemma 2. The mapping $D$ defined in Eq. (8) is an affine homeomorphism, i.e. $D$ is affine and

i) injective,

ii) continuous with respect to the weak topologies, and

iii) if $\left\{\mu_{n}\right\}$ is a sequence in $M_{+}^{1}\left(\mathbb{R}^{2}\right)$ such that $D\left(\mu_{n}\right) \rightarrow W \in S(H)$ weakly, then there exists a uniquely determined element $\mu \in M_{+}^{1}\left(\mathbb{R}^{2}\right)$ such that $\mu_{n} \rightarrow \mu$ weakly and $W=D(\mu)$.

Proof.

i) Assume $D\left(\mu_{1}\right)=D\left(\mu_{2}\right)$ where $\mu_{1}, \mu_{2} \in M_{+}^{1}\left(\mathbb{R}^{2}\right)$. For all $(x, y) \in \mathbb{R}^{2}$ this implies that

$$
\left(\psi(x, y), D\left(\mu_{1}\right) \psi(-x,-y)\right)=\left(\psi(x, y), D\left(\mu_{2}\right) \psi(-x,-y)\right) .
$$

From Eq. (12) we infer that

$$
\hat{v}\left[\mu_{1}\right](x, y)=\hat{v}\left[\mu_{2}\right](x, y)
$$

holds for all $(x, y) \in \mathbb{R}^{2}$. From the uniqueness of characteristic functions $v\left[\mu_{1}\right]=$ $v\left[\mu_{2}\right]$ follows which, in turn, implies $\mu_{1}=\mu_{2}$ as the density (14) is strictly positive for all $(x, y) \in \mathbb{R}^{2}$.

ii) Under the duality $L^{1}(H) \times L(H) \in(T, A) \rightarrow \operatorname{tr}(A T)$ the topological dual of $L^{1}(H)$ can be identified with $L(H)$. Given a sequence $\left\{D\left(\mu_{n}\right)\right\}$ in $C S(H)$ with $\mu_{n} \rightarrow \mu$ weakly, for any continuous linear functional $f$ on $L^{1}(H)$ there exists an uniquely determined element $A_{f} \in L(H)$ such that

$$
\begin{aligned}
n)) & =\int d \mu_{n}(x, y) f(P(x, y))=\int d \mu_{n}(x, y) \operatorname{tr}\left(A_{f} P(x, y)\right) \\
& =\int d \mu_{n}(x, y)\left(\psi(x, y), A_{f} \psi(x, y)\right) .
\end{aligned}
$$

As $(x, y) \rightarrow\left(\psi(x, y), A_{f} \psi(x, y)\right) \in C_{b}\left(\mathbb{R}^{2} ; \mathbb{C}\right)$ we conclude that

$$
f\left(D\left(\mu_{n}\right)\right) \rightarrow f(D(\mu))
$$

for all linear continuous functionals on $L^{1}(H)$.

iii) As $D\left(\mu_{n}\right) \rightarrow W \in S(H)$ weakly we have for all $A \in L(H)$

$$
\lim _{n \rightarrow \infty} \operatorname{tr}\left(D\left(\mu_{n}\right) A\right)=\operatorname{tr}(W A) .
$$

Setting $A=P(x, y)$, for all $(x, y) \in \mathbb{R}^{2}$ we obtain by means of Eq. (20)

$$
\lim _{n \rightarrow \infty}\left(g * \mu_{n}\right)(x, y)=\frac{1}{\pi} w(x, y) .
$$

Accordingly, in the weak topology

$$
\lim _{n \rightarrow \infty} \rho_{D\left(\mu_{n}\right)}=\rho_{W} .
$$

This implies (cf. e.g. [5])

$$
\lim _{n \rightarrow \infty} \hat{\gamma}(x, y) \hat{\mu}_{n}(x, y)=\hat{\rho}_{W}(x, y)
$$


for all $(x, y) \in \mathbb{R}^{2}$. This is equivalent to the fact that for all $(x, y) \in \mathbb{R}^{2}$,

$$
\lim _{n \rightarrow \infty} \hat{\mu}_{n}(x, y)=(\hat{\gamma}(x, y))^{-1} \hat{\rho}_{W}(x, y)
$$

Since the mapping

$$
(x, y) \rightarrow(\hat{\gamma}(x, y))^{-1} \hat{\rho}_{W}(x, y)
$$

is continuous and normalized to 1 in $(0,0) \in \mathbb{R}^{2}$ we eventually obtain, using Theorem 48.7 II of ref. [5], that i) there exists an uniquely determined element $\mu \in M_{+}^{1}\left(\mathbb{R}^{2}\right)$ such that $\mu_{n} \rightarrow \mu$ weakly, and ii)

$$
\hat{\mu}(x, y)=(\hat{\gamma}(x, y))^{-1} \hat{\rho}_{W}(x, y)
$$

for all $(x, y) \in \mathbb{R}^{2}$.

It remains to show that $W=D(\mu)$. But this is obvious from Lemma 1 as we have shown that $\hat{\sigma}_{W}=\hat{\mu}$ is a characteristic function and from the uniqueness part of this lemma. This ends the proof of Lemma 2.

Our central result is contained in the following theorem.

Theorem. Let $\mathrm{CS}(H)$ be the convex set of classical states of the harmonic oscillator, then

i) $C S(H)$ is a simplex and the set of extreme points is the set of coherent states

$$
\operatorname{ex}(C S(H))=\left\{P(x, y) ;(x, y) \in \mathbb{R}^{2}\right\}
$$

ii) $\mathrm{CS}(H)$ is the closed convex hull of its extreme points

$$
C S(H)=\operatorname{cl}(\operatorname{con}(\operatorname{ex}(C S(H))))
$$

where the closure refers to the weak topology of the Banach space $L^{1}(H)$,

iii) each $D(\mu) \in C S(H)$ is the barycenter of an uniquely determined probability measure $\pi_{\mu}$ on $L^{1}(H)$ (equipped with the weak Borel $\sigma$-field) satisfying $\pi_{\mu}(\operatorname{ex}(C S(H)))=1$, i.e.

$$
\operatorname{tr}(A D(\mu))=\int_{\operatorname{ex}(C S(H))} d \pi_{\mu}(T) \operatorname{tr}(A T)
$$

holds for all $A \in L(H)$.

Proof.

i) Due to the fact that $M_{+}^{1}\left(\mathbb{R}^{2}\right)$ is a simplex the same holds for $C S(H)$ as, according to Lemma 2, part i) the mapping $D$ is an affine bijection and preserves, therefore, order properties. Accordingly, the set $\operatorname{ex}(C S(H))$ is the image of $\operatorname{ex}\left(M_{+}^{1}\left(\mathbb{R}^{2}\right)\right)=$ $\left\{\delta_{(x, y)} ;(x, y) \in \mathbb{R}^{2}\right\}$ under this mapping where $\delta_{(x, y)}$ denotes the Dirac measure on $\mathbb{R}^{2}$ concentrated at $(x, y) \in \mathbb{R}^{2}$. From this (38) follows.

ii) Firstly, we prove that

$$
\int d \mu(x, y) P(x, y) \in \operatorname{cl}\left(\operatorname{con}\left(\left\{P(x, y) ;(x, y) \in \mathbb{R}^{2}\right\}\right)\right)
$$

for all $\mu \in M_{+}^{1}\left(\mathbb{R}^{2}\right)$. Suppose that there is a $\mu \in M_{+}^{1}\left(\mathbb{R}^{2}\right)$ such that $D(\mu)$ does not satisfy (41). The geometric version of the Hahn-Banach theorem then ensures the existence 
of a strictly separating hyperplane, i.e. there exists a selfadjoint $A \in L(H)$ and a real number $\alpha$ such that

$$
\operatorname{tr}(A D(\mu))=\int d \mu\left(x^{\prime}, y^{\prime}\right) \operatorname{tr}\left(A P\left(x^{\prime}, y^{\prime}\right)\right)<\alpha \leqq \operatorname{tr}(A P(x, y))
$$

for all $P(x, y) \in L^{1}(H),(x, y) \in \mathbb{R}^{2}$. Integration yields

$$
\int d \mu\left(x^{\prime}, y^{\prime}\right) \operatorname{tr}\left(A P\left(x^{\prime}, y^{\prime}\right)\right)<\alpha \mu\left(\mathbb{R}^{2}\right) \leqq \int d \mu(x, y) \operatorname{tr}(A P(x, y))
$$

which is a contradiction.

Accordingly, we have

$$
\operatorname{con}\left(\left\{P(x, y) ;(x, y) \in \mathbb{R}^{2}\right\}\right) \subset C S(H) \subset \operatorname{cl}\left(\operatorname{con}\left(\left\{P(x, y) ;(x, y) \in \mathbb{R}^{2}\right\}\right)\right) .
$$

By part iii) of Lemma 2 the set $C S(H)$ is weakly closed such that (39) follows if we take into account Eq. (38).

iii) The mapping $\Phi$ defined in Eq. (6) is continuous and therefore Borel measurable. For any $D(\mu) \in C S(H)$ the probability measure $\pi_{\mu}=\Phi \circ \mu$, the image of $\mu$ under $\Phi$, satisfies for all $A \in L(H)$

$$
\operatorname{tr}(A D(\mu))=\int d \mu(x, y) \operatorname{tr}(A P(x, y))=\int_{L^{1}(H)} d \pi_{\mu}(T) \operatorname{tr}(A T) .
$$

As ex $(C S(H))$ is weakly closed and therefore measurable, we obtain

$$
\pi_{\mu}(\operatorname{ex}(C S(H)))=1,
$$

which proves the existence of the integral representation (40). Uniqueness of this integral representation follows from the uniqueness part of Lemma 2 together with the property that $\Phi$ considered as a mapping from $\mathbb{R}^{2}$ onto $\operatorname{ex}(C S(H))$ is a homeomorphism which, in turn, follows from Lemma 2 where we also need part ii). This ends the proof of the theorem.

\section{Remarks}

1. In the present situation an application of classical Choquet theory is not possible as $C S(H)$ is the homeomorphic image of the non-compact set $M_{+}^{1}\left(\mathbb{R}^{2}\right)$. Nevertheless, part i) and part iii) of the theorem are equivalent.

This is due to the fact that the separability of $H$ implies that $L^{1}(H)$ is a separable dual space which according to [4, Th. III. 3.1.] has the $R N$-property. We therefore can use the results of $[6,7]$ which contain an exact analogue of the classical existence and uniqueness theorems for barycentric integral representations of closed and bounded convex subsets of a Banach space with $R N$-property.

2. The coherent state vectors are intimately connected with the (trivial) BoseFock-space structure of the Hilbert space of the harmonic oscillator (cf. [3]), namely

$$
H=F_{+}(\mathbb{C}) .
$$

A generalization of our results to the simplex structure of the classical states on $F_{+}\left(\mathbb{C}^{k}\right), k \in \mathbb{N}$, is quite straightforward. Technical difficulties, due to our method and obviously not specific to the problem, arise in the case of the boson field with state 
space $F_{+}(K), K$ an infinite dimensional separable complex Hilbert space, by virtue of the fact that on $K$ there exists neither an analogue of the Lebesgue measure nor a rotation invariant Gaussian measure.

3. It is well known that $S(H)$ is a convex set with extremals (pure states)

$$
\operatorname{ex}(S(H))=P(H),
$$

where $P(H)$ denotes the set of orthogonal projections onto one-dimensional subspaces of $H$.

This fact implies

$$
\operatorname{ex}(C S(H))=C S(H) \cap \operatorname{ex}(S(H))
$$

and shows that the coherent states are the only pure states which are representable according to our definition.

4. A convenient possibility to characterize the fundamental difference between a system in classical mechanics and quantum mechanics is provided by the structure of the convex set of states of these systems. Whereas the state space of a classical system, the set of all probability measures on its phase space is always a simplex, the state space of a quantum system, here $S(H)$, is never a simplex. This is due to the fact that for any $W \in S(H), W \notin P(H)$, there exist (infinitely many) different representations of the form

$$
W=\sum_{i} p_{i} P_{i},
$$

where $P_{i} \in P(H), p_{i} \geqq 0, \sum_{i} p_{i}=1$, (cf. e.g. [8]).

Considering the quantum harmonic oscillator in a pure state, it is well known that only in a coherent state the quantum harmonic oscillator exhibits a structural analogy to the classical harmonic oscillator. In this work we have shown that, in addition, the closed convex hull of these states has a structure which is characteristic for a classical system.

\section{References}

1. Glauber, R. J.: Photon statistics. In: Cohen, E. G. D. (ed.) Fundamental problems in statistical mechanics. Amsterdam: North-Holland 1968, pp. 140-187

2. Klauder, J. R., Sudarshan, E. C. G.: Fundamentals of quantum optics. New York: Benjamin 1968

3. Davis, E. B.: Quantum theory of open systems. London: Academic Press 1976

4. Diestel, J., Uhl, J. J. Jr.,; Vector measures. Providence, RI: AMS 1977

5. Bauer, H.: Wahrscheinlichkeitstheorie, 2nd (ed.) Berlin: De Gruyter 1974

6. Edgar, G. A.: A noncompact Choquet theorem. Proc. Am. Math. Soc. 49, 354-358 (1975)

7. Bourgin, R. D., Edgar, G. A.: Noncompact simplexes in banach spaces with the Radon-Nikodym property. J. Funct. Anal. 23, 162-176 (1976)

8. Beltrametti, E. G., Cassinelli, G.: The logic of quantum mechanics. Reading M. A.: Addison-Wesley 1981

Communicated by G. Parisi

Received February 5, 1986 